\title{
The Use of Human Body as a Medium in Architectural And Structural Education: "Yogarch" Program
}

\author{
Sebla Arın Ensarioğlu \\ 1 Assist. Prof Dr., Faculty of Architecture, Bursa Uludağ University, Bursa, Turkey. (Principal contact for editorial \\ correspondence), Email: seblaarin@uludag.edu.tr
}

\section{Abstract Purpose}

One of the main difficulties for first year architecture students is to sort out complicated physical rules of constructional systems and reflecting those rules to their designs. These topics are also difficult to understand in built environment education (B.E.E.) designed for children. In order to simplify the learning process of these abstract topics, an experiential program is designed and explained in the scope of this paper. This paper aims to introduce the YogArch program and the outcomes of its implementation.

\section{Design/Methodology/Approach}

This program is based on two different disciplines, which are yoga and architecture. Therefore, it is called "YogArch". YogArch matches specific yoga asanas (positions) with suitable architectural elements and systemic principles. This program gives the participant a chance to experience these constructional facts on his/her own body. In the scope of this paper 8 different implementations of YogArch program are examined. There are two different participant groups: children and first year undergraduate architecture students. There are totally 221 participants in these two focus groups. In the scope of this research, the outputs of the workshops are collected and analyzed, findings are generated and some suggestions are made on the future use of the program.

\section{Findings}

The outcomes of implemented YogArch workshops are analyzed. According to these findings, this program supports creating built environment awareness for children. For the undergraduate students, the program supported and clarified the knowledge they've obtained in their formal courses. Also, it is a practical method for simplifying complicated terms in the curricula.

\section{Research Limitations/Implications}

The YogArch program is based on practicing some yoga poses in order to create physical experience. During the implementation of the workshop schedule, some participants had difficulties due to their own lack of training, in practicing some of the poses which require reasonable amount of strength. In such cases the instructor had to support and direct the participant to practice the pose in a more suitable version and develop body awareness.

Practical/Social Implications

The safety of a living environment depends on the consciousness level of inhabitants and users. Providing sufficient knowledge on constructional principles of buildings for the entire society and extending built environment education have vital importance especially for the countries which are at risk about earthquakes, like Turkey.

Originality/Value

This study focuses on offering an experimental education model in structural and architectural design education for all ages. This paper introduces a new educational program called YogArch. The proposed model differs from previous studies, as it expands the educational methods in formal and informal architectural education.

Keywords: Architectural education, yoga and architecture, structural design education, built environment education, body experience 


\section{INTRODUCTION}

From the beginning of architectural history, human body has been an inspiration for architectural design both in metaphorical and morphological terms. Beginning with Vitrivius in Roman Architecture, human body became the main source of proportional systems in structural design. As far as human body was believed to be perfect structurally and aesthetically, architects tried to reflect this harmony to their buildings. In Renaissance, which might be called as rediscovery of Antiquity, human body became a template for designing not only a single building, but also the city as a whole. In the following periods, as there were profound examinations on the mechanics of human body, the constructional principles of human body leaded a way for the creations in architectural and industrial design (Plowright, 2018). In the 19th and 20th centuries the need of standardization in architecture, caused to emerge measurement systems based on the size of human body and its limbs.

Designers have been inspired from nature in architecture and engineering for many centuries. Therefore, examining biological/natural systems is an enlightening approach for understanding the mechanical solutions and structural systems of the design-work created by humanbeings. The YogArch program examined in terms of this paper, considers human body as an instrument for understanding architectural elements and principles of constructional systems. If human body is an inspirational source for architecture, it might also be used as a handy material for revealing architectural physiology.

The use of human body in architectural education became a popular approach in recent years, especially in first year undergraduate education and built environment education for children. Exploring the human body and focusing on the differences created by the changes in body posture under several forces, helps to clear up the fundamentals of structural systems. Based on this assertion, a program called YogArch is designed. It matches some yoga asanas (positions) with some architectural elements and systemic principles. This program gives the participant a chance to experience these constructional principles on his/her own body. The case of self-experimentation eases and shortens the participant's perception process on this subject. This can be a practical alternative to conventional educational systems which are based on theoretical explanations of complicated physical rules.

This paper aims to clarify the origin, the methodology and potentials of YogArch program. The historical background of using human body in experiencing architecture will be discussed in the literature review. The YogArch schedule, the structured workshops and the collection process of the outputs of these workshops will be explained in the methodology section. In the evaluation and discussion part, the results of the workshops will be examined. Finally, in the conclusion, some suggestions will be made on the potential use of this program in formal and informal architectural education.

\section{HUMAN BODY IN EXPERIENCING ARCHITECTURE}

Human body, which is a system of proportional relations, is also a source of defining ratios and measurements in architectural design. The anthropometric measurements of human body are important in defining architectural standards. On the other hand, the complicated mechanisms 
The Use of Human Body as a Medium in Architectural And Structural Education: "Yogarch" Program

which keep the human body erected, and working principles of these systems inspired the disciplines of architecture and engineering in structural terms. All of these various aspects about the relation between human body and architecture will be reviewed in this section.

\section{Morphological interaction between human body and architecture}

Making an analogy between human physiology and structural elements of the building is a traditional approach in architecture. In Ancient Rome, Vitrivius stated that in the design of an aesthetically perfect temple, the proportions of human body can be used as a pattern (Vitrivius, 1960). He identifies the order of columns, by which he clarifies the relationship between human body and building. According to Vitrivius each type of column (Doric, Ionic, and Corinthian) is matched with a different type of body (male, female and girl) (Figure 1) (Plowright, 2018).

Figure 1. Column typehuman body type matchings (Url-1)

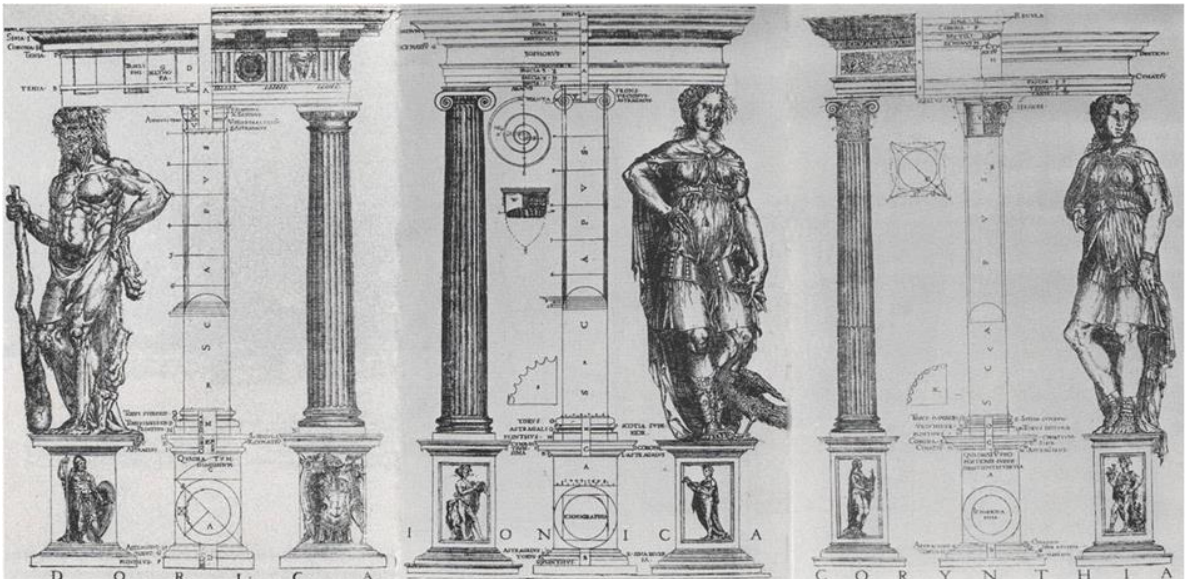

Alberti, connected human body references to building elements in Renaissance. He established analogies between human body and building elements stating that "with every type of vault, we should imitate Nature throughout, that is, bind together the bones and interweave flesh with nerves running along every possible section" (Alberti, 1988; Plowright, 2018).

Another Renaissance architect, Francesco di Giorgio, created an analogy between human body and a church plan (Figure 2). He also stated that there is a resemblance between a column capital and a human face (Figure 3). He mentioned the importance of using the proportions of human body in architectural design in his book: "Trattato di Architettura Civile e Militare" written in 1470 (Steadman,1979).

According to Filarete, a Florentine architect lived in 15th century, the limbs and cavities of human body is similar to the building which has voids such as doors and windows. Furthermore, Filarete believed that a building or a city is a mortal creature like a human-being which lives, gets ill, recovers and dies at the end (Vidler, 1990). 


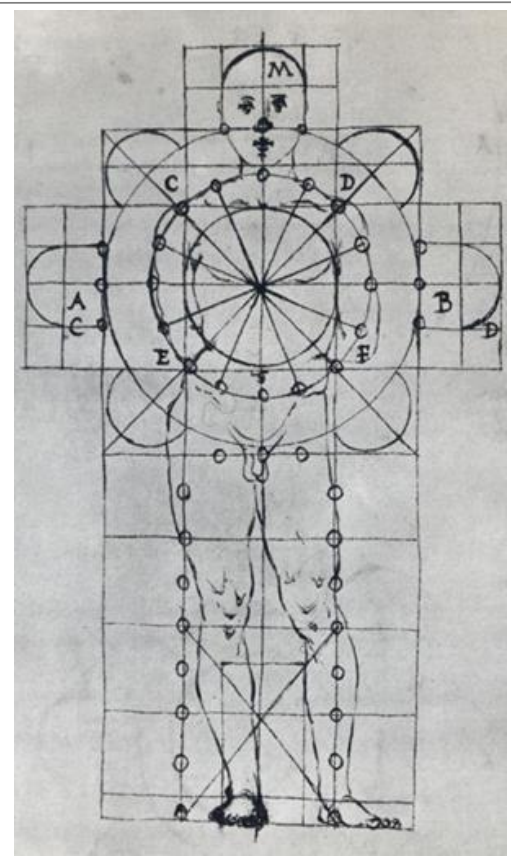

Figure 2. Proportional rules for the construction of a church ( Url-2)

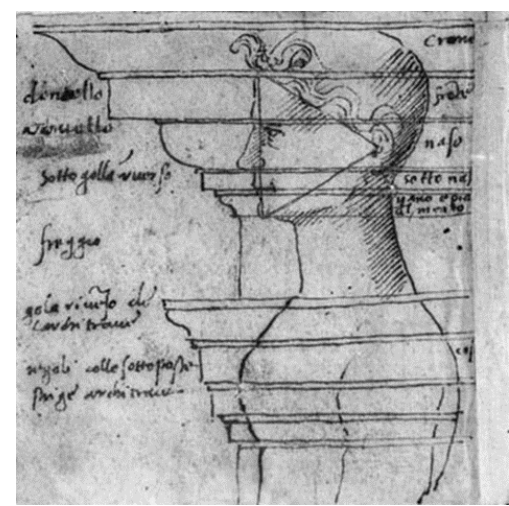

Figure 3. Proportional rules for the construction of a column capital (Url-3)

Leonardo da Vinci, a pioneer architect and artist of Renaissance, drew the Vitruvian Man based on "De Architectura" written by Vitrivius. Vitrivius wrote the oldest known book of architecture, between 30 and 15 BC. Fifteen centuries later in 1492, Da Vinci created the most famous visual material of architectural history and immortalized the analogy between the geometry of "perfect" proportions of human body and architecture (Figure 4). 
The Use of Human Body as a Medium in Architectural And Structural Education: "Yogarch" Program

Figure 4. Vitruvian Man by Leonardo Da Vinci (Url-4)

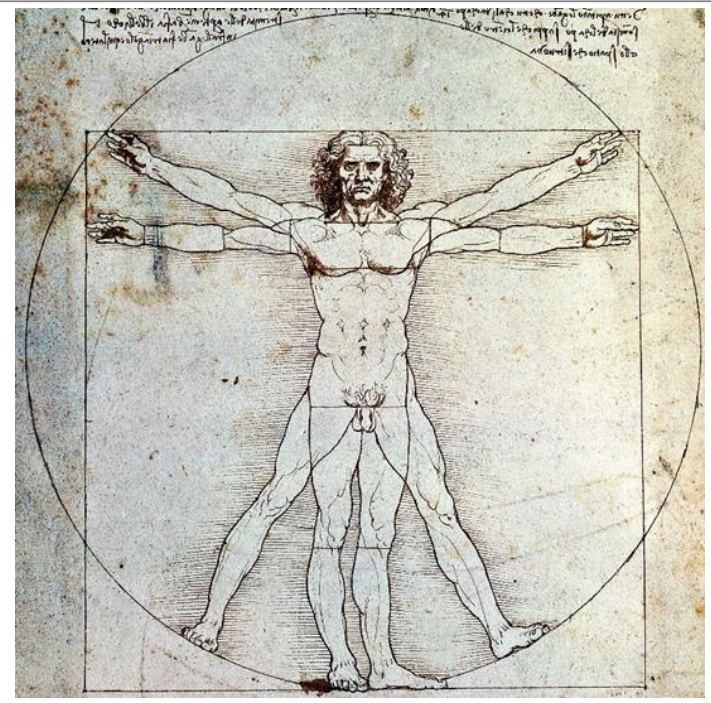

Vidler (1990), described the history of the body in architecture as the progressive distancing of the body from the building; a gradual extension of the anthropomorphic analogy to the final 'loss' of the body as an authoritative foundation for architecture, starting from Vitruvius to contemporary architecture. He classified this process into three stages: the building as body; the building exemplifying bodily states; the environment carrying bodily characteristics. In Vitruvian and Renaissance theory, the body is seen as a perfect source of inspiration for designing buildings. But the modernist thought is more focused on the concept of body rather than just the plain morphology of it. An exceptional representative of morphological analogy between human body and architecture in modernity is Le Corbusier and the scale system he created: "Modular". Le Corbusier used the measures of human body, indeed his own body, in order to establish a proportion system. "Modular" was first published in 1950. In his book, Corbusier used the principle of the Golden Section in order to derive two series of dimensions from the human figure. First one is based on the height of a standing man with upraised arm: 2.26 meters and the second one is based on the height of this man measured from his feet to the top of his head: 1.83 meters (Figure 5) (Le Corbusier, 2014).

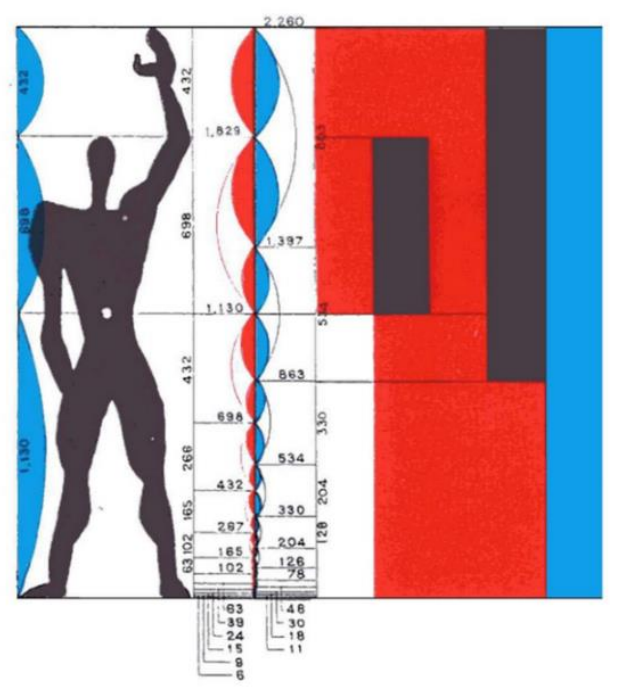

Figure 5. Modular by Le Corbusier (Url-5) 
The tradition of the anthropomorphic analogy between human body as the nature's creation and architectural building as human-being's creation constitutes a wide literature, a limited part of which is mentioned in the previous paragraphs. This analogy might be accepted as a medium for understanding the logic of the systematic rules of proportion, scale and structure in architectural buildings and elements.

\section{Technical interaction between human body and architecture}

The human body is also an inspiration for architecture in terms of its mechanical and technical integrity. The biological mechanisms of natural creations achieved a significant breakthrough in design sector in recent years. The bionic design innovation in building sector leads up reasonable use of intelligent technology and sustainable development in architecture (Lotfabadi, Alibaba \& Arfaei, 2016). The term "bionic" became prevalent in 20th century. It is an interdisciplinary concept which combines biological sciences with various fields such as construction, architecture, engineering, medicine, material and etc. (Chiou \& Chiu, 2010). Bionics might be accepted as a bridge between nature and technology. As far as the nature is based on sustainable development of complex systems, it might bring new initiatives to innovative constructional systems.

Another term which engages biology and architecture is "biomechanics". Biomechanics is the study of the movements of living things using the science of mechanics (Knudson, 2007). Understanding the kinesiology of human body might be useful for designing adaptive high-technology constructive systems.

The interaction of human body (as a biological form) with structural design is a relatively new concept, which has a big potential for the future of architecture. Since the biological qualities of the human body can be a resource for structural system design, it can also be a resource for perceiving the working principles of existing structural systems.

\section{METHODOLOGY}

As explained in the previous chapter, human body has a rich potential for shaping and perceiving architecture. This reveals the educative potential of human body. In order to use this potential, a workshop schedule that might be used in structural design education in architecture, is designed and it will be explained in the scope of this paper. This program combines two disciplines and it is called "YogArch", taking its name from the disciplines it is based on: yoga and architecture.

One of the main difficulties for first year architecture students is to sort out complicated physical rules of constructional systems and reflecting those rules to their designs. Their limited experience in the field of architecture makes it more difficult to analyze the structural rules of three dimensional design. During architectural and basic design courses and theoretical physics lectures the freshmen students are introduced to these academic subjects. But this knowledge might become much more permanent if supported with experimental techniques. On the other hand, architectural education shouldn't be admitted only in vocational terms. It should be perceived as a life-long journey and ought to be supplied to each member of the society regardless of their age and profession. But it might be an abstract subject for a child or a nonprofessional to understand how buildings erect on earth and how structural systems work. The "YogArch" program is designed to make it 
The Use of Human Body as a Medium in Architectural And Structural Education: "Yogarch" Program

simpler for both children and adults to internalize the rules of structural systems. The resemblance between structural principles of the human body and the architectural construction is an inspiration for this program. Human body is a handy tool for an individual to experience the basic forces in a constructional system. The skeleton and the muscular system together can be instructive for comprehending the laws of balance. The mental effort to conceive structural principles of architectural elements should be supported with physical perception. The need of selfexperimentation inspired to use a physical method during which an individual can sense the changes happening in his/her own body and relate these with constructional rules. With the aim of designing a selfexperimental program, yoga was chosen as a medium. The main forces and architectural elements are matched with yoga postures in the context of this physical workshop. These workshops were realized with two different target groups: children aged between 6-14 years and first year architecture students. The aim was to reveal the effects of bodyexperience in structural education. Also the differing perceptions of the participants depending on their ages and the academic education level they have was another subject that was searched for. The duration of the workshops was decided according to the concentration level of the participant group. The findings of the implemented workshops were collected by various methods including, self-observation, questionnaires, interviews and group conversations. Also visual recordings were also taken under information and permission of the participants. The collected data was examined and analyzed in order to systemize the effects of the program on the participants. The general inclination of the participant groups was visualized by graphics based on the findings. At the end, depending on the analyses, some suggestions were developed on the use of YogArch workshops and the potentials of the program. The details of the methodology are explained in this section.

\section{The Structure of YogArch Workshops}

Yoga is an ancient discipline combining sensational and physical experience, in which the individual searches his/her own limits. In the physical practices of yoga some poses, which are called "asana", are used. These poses put human body into specific postures. They are based on three topics: flexibility, balance and power. By practicing these poses, one can explore the working principles of architectural elements and systems on his/her own body. In other words, yoga asanas might be assumed to be practical tools for comprehending physical laws of balance.

YogArch program is constituted from a set of yoga asanas supported by visual materials. The set is designed in the norm of an ordinary beginnerlevel yoga class. The order of the asanas is not coincidental. The program consists of three parts respectively: simple warming-up asanas, more complicated asanas of balance \& power and relaxation poses; following the order of a typical yoga practice. The methodology of the program is based on three sections: audiovisual learning, physical experience and evaluation. The program is beneficial for both children and adults such as students of architecture. As far as there is a wide range of participants, the content of the audio-visual part is changeable depending on the needs and the expectations of the participant group. As it will be explained in the following parts, YogArch program might be practiced as a single workshop for children as a part of built environment education; also as a 
warm-up exercise for architectural design course or a hands-on learning exercise for structural design course in first year educational schedule of undergraduate architecture education.

A YogArch workshop longs for approximately 60-80 minutes, depending on the age level of the participant group. The program consists of 22 different asanas each of which symbolizes a constructive principle or an element. Some of these asanas practiced alone, some of them with a group. Before practicing the pose, the instructor, preferably experienced both in architecture and yoga, explains and gives adequate information on visual materials, about the architectural element or principal that will be practiced. Following this brief explanation, the instructor practices the asana him/herself (if needed getting help from a participant) and explains the key-points that should be focused on during the implementation of the pose. The tensile and pressure forces felt on body are matched with the forces in the constructional system. Therefore, the participants focus on the changes on their own body when they are in the pose, so that they can understand the similar situation in a building. At the end of each workshop the participants are asked to answer a short questionnaire in order to get feedback about the pros and cons of the program. Also face to face interviews and group evaluation are the methods used for collecting participants' opinions. Photos are taken for recording the flow of the program. The participation to the workshops are on voluntary basis. All of the documentation are made under the information and permission of the participants and their parents (in case of children attendees).

\section{YogArch Schedule}

The schedule consists 22 different yoga poses. Each pose is matched with an architectural element or a principle. In this section these matchings will be explained in detail. All poses will be explained according to the implementation order. First the name of the architectural element/system, then the original (Sanskrit) name of the pose and then the English name of the pose will be given. The working principles of the element and matching qualities of the posture and the element will also be explained (Arın, 2013; Pappas, 2006; Kaminoff \& Matthews, 2012; Worby, 2007). The postures and the elements they are matched with are also shown in Figure-6. 
The Use of Human Body as a Medium in Architectural And Structural Education: "Yogarch" Program

Figure 6. YogArch Schedule Diagram

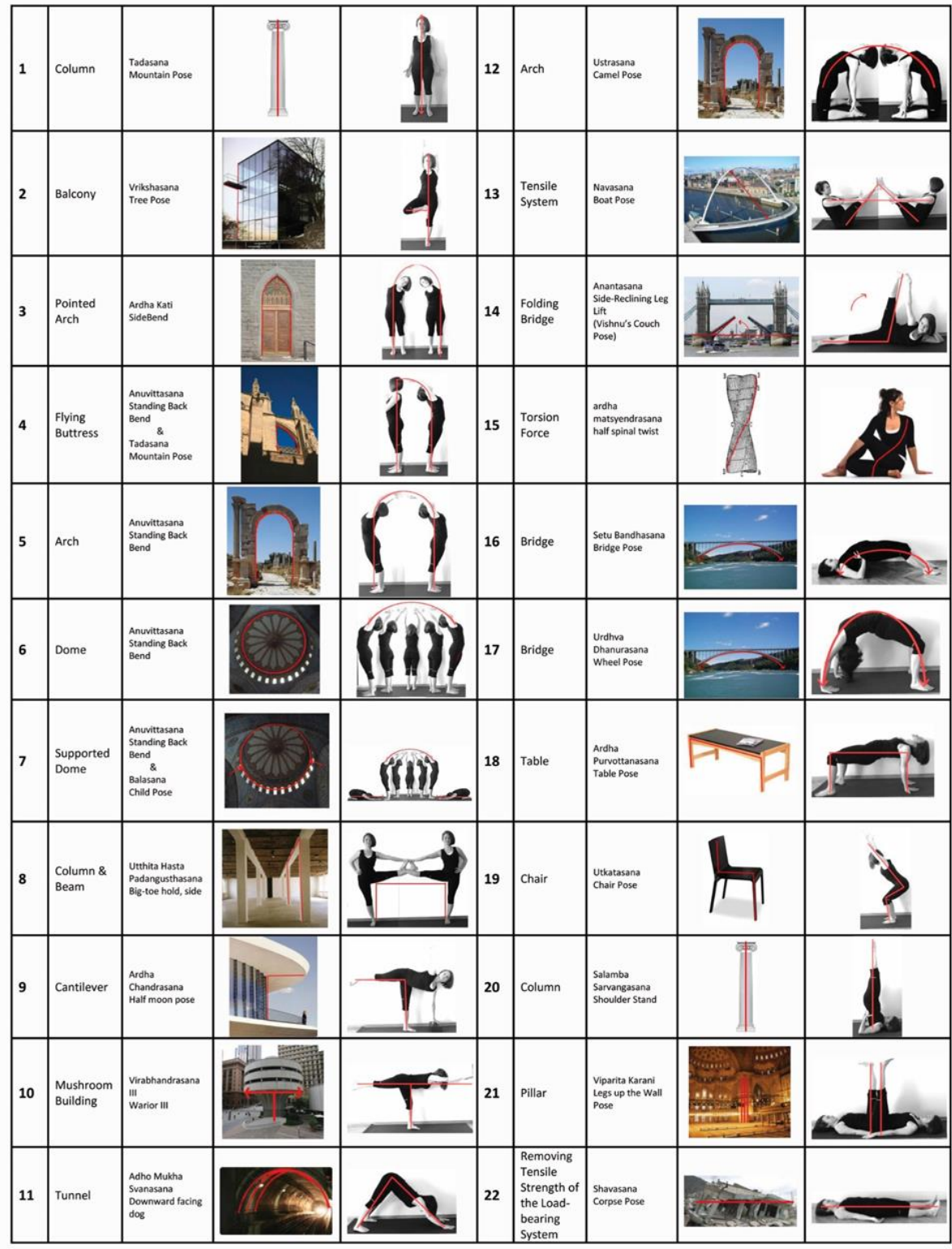

1. Column / Tadasana / Mountain Pose: The column is one of the main structural elements in buildings. In this program it is symbolized with the natural posture of human body, which is called "mountain pose". In this pose, the person stands hanging his/her arms besides the torso, opens the legs as wide as the hips and balances the body weight on the feet. The spinal column bears the load of the body and transfers it to the ground. Similarly, a column bears the load of the building and transfers it to the ground through foundation.

2. Balcony / Vrikshasana / Tree Pose: In this pose the person places one foot, high up on his/her thigh on the other side. This pose replicates the balanced stance of a tree. The human body stands balanced on one leg while the other one is twisted from the knee and attached to the standing leg. In the case of a balcony, the floor slab of the balcony is attached to the main building and transfers the load from that junction point.

3. Pointed Arch / Ardha Kati Chakrasana / Side Bend: In this pose the person stretches his/her body and leans to a side by extending his/her arm over the head. The tension force on the lengthened side of 
the body is deeply felt. When this pose is practiced with a partner catching the leaning hand, it resembles a pointed arch. The hands of the partners support each other and work together like a key stone. The person might experience the similar forces of an arch structure on his/her body.

4. $\quad$ Flying Buttress / Anuvittasana \& Tadasana / Standing Back Bend \& Mountain Pose: The combination of these two poses practiced by two partners is used to visualize a load-bearing element called flying buttress which is generally used in medieval buildings. The inclined bended structure transfers the lateral forces that push a wall outward to the ground while creating a void between two elements. The secondary element is attached to the main building from an upper level, so that the structure is much lighter by creating a gap.

5. Arch / Anuvittasana / Standing Back Bend: This is a couple pose which is realized by two partners siding back and it resembles the arch structure. The compression force on the back of the partners lets them experience the forces that help to protect the integrity of an arch.

6. Dome / Anuvittasana / Standing Back Bend: This pose is another version of Pose- 5 by at least six people. It is used in order to visualize the dome structure which is a circular covering element. Each member of the group works like a rib of the dome. In a dome structure, the compression force on top is transferred to the main building by the ribs creating a tension force. The group members might feel the similar forces on the meeting point of their hands and on their stretched torso. In order to deepen the pose, the instructor might apply some load on the top of the group members' hands. By this way the practitioners might understand how the load on a dome is transferred to the ground by the structural system. This structure might also be formed by practicing standing front bend.

7. Supported Dome / Anuvittasana \& Balasana / Standing Back Bend $\&$ Child Pose: If the base diameter of a dome is relatively huge, a support might be needed. Also in case of a back bend (or a front bend), if the distance between the toes and finger tips, which are the start and end points of the arch, is lengthened it becomes difficult to keep the body steady because of the compression force. In this case a support from the feet might be useful to avoid collapsing and stay in the pose for a longer period. By this way the members of the group can understand the function of the supporting elements. In order to make all members understand the forces, the ones practicing back or front bend and the ones practicing child pose might exchange their positions.

8. Column \& Beam / Utthita Hasta Padangusthasana / Big-toe Hold, Side: In this pose, two individuals are holding up their legs meanwhile holding their partners' leg with their hands and supporting each other to stand stable. This is a good example for understanding the column-beam working principle. The legs on the ground symbolize columns and the legs together in the air symbolize the beam. When the members of the couple stand closer to each other and the distance between their bodies is shorter, they can keep their stability for a longer period. Also when they put their legs onto each other's, it becomes more durable against a force implemented on the unity of two legs. Similarly, in a column and beam system the length and width of a beam between two columns determines the strength of the system.

9. Cantilever / Ardha Chandrasana / Halfmoon Pose: In this pose one leg is held up to $90^{\circ}$ angle position to the other. Meanwhile, the other 
The Use of Human Body as a Medium in Architectural And Structural Education: "Yogarch" Program

leg and one arm are touching the ground for supporting the body. This position is proper for perceiving the logic of cantilever. In this position the arm and the leg attached to the ground symbolize the columns of the building, where as the torso symbolizes the floor slab between the columns and the leg up in the air symbolizes the slab extended. The instructor might apply force on different parts of the leg in the air. This way the practitioner might understand that the closer the force to the center of gravity, the more durable is the system. If the force is implemented on the foot, the person cannot stay steady as much.

10. Mushroom Building / Virabhandrasana III / Warrior III: In this pose the person stands on only one leg. Meanwhile, the other leg and arms stay up, parallel to the floor. This posture resembles the look of a mushroom building in which there is a central load-bearing element. If some force is applied on the hands and the foot up, the experience might become deeper.

11. Tunnel / Adho Mukha Svanasana / Downward Facing Dog: All members of the group are aligned side by side practicing "downward facing dog" pose. In this pose the hand and the foot are on the ground, forming a void under the hip. This position resembles the tunnel structure which might be explained as a linear repetition of arch. After forming the tunnel structure, the participants might pass under it one by one.

12. Arch / Ustrasana / Camel Pose: This position is the combination of two individuals practicing "camel pose" back to back. In this pose each individual stands on his/her knees, bending backwards getting support from the arms attached to the heels. Outer contour of the couple is another version of visualizing the arch structure. The camel pose also resembles flying buttress, when practiced individually.

13. Tensile System / Navasana / Boat Pose: This is a couple pose. When the boat pose is practiced with a partner, it creates a tension on the arms of both practioners. In the boat pose, the person tries to stay stable on his/her hips on the ground, while the legs are up and the arms are pointing forward. If the soles of feet and the hands of the partners are attached together it becomes easier to stay steady. The changes in the distance between the partners effect the tension force felt by them.

14. Folding Bridge / Anantasana / Side-Reclining Leg Lift (Vishnu's Couch Pose): In this exercise the person holds up/down his/her leg while lying on one side. The femur is attached to the ilium by hip joint and the movement of femur is realized by this joint and the muscles. The leg moving upwards and downwards from the hip joint is a good example of animating the opening/closing action in folding bridges.

15. Torsion Force / Ardha Matsyendrasana / Half Spinal Twist: In this pose the person rotates his/her spine on opposite directions trying to keep the coccyx steady. Each vertebra rotates at different angles depending on the distance from the coccyx. This pose is included in the program in order to explain torsional force. Structural systems have to resist against tore caused by constructive and natural forces. The elasticity of each person is different so that the maximum angle they are able to turn also differ. Similarly, the elasticity of materials and the constructional system used in the building changes its resistance to torsional force.

16. Bridge / Setu Bandhasana / Bridge Pose: In this pose the body is bended like a bow with the support it gets from the feet $\&$ the shoulders. 
These two parts of the body are the only points that the body is attached to the ground and transfers the load. This pose helps to perceive the constructional principles of a bridge which crosses a long distance with the support from two edges.

17. Bridge / Urdhva Dhanurasana / Wheel Pose: This pose is similar to Pose-16, but in this one the support is coming from the feet $\&$ hands. It is another way of visualizing the bridge structure. The person practicing these poses one after the other, might experience the difference created in the tension and compression forces with the change in the curve of an arch.

18. Table / Ardha Purvottanasana / Table Pose: This pose is the reflection of an everyday object: table. The posture of the body resembles a panel parallel to the floor standing on four support points. The torso facing upwards symbolizes the top whereas the legs and arms symbolize four legs of the table. The instructor might direct the individual to take off his limbs in the air to experience the change in the center of gravity because of the missing supports. Different positions such as one limb up, the cross limbs up and two limbs on one side up create different structural experiences.

19. Chair / Utkatasana / Chair Pose: Another popular everyday object is chair. This pose gives a chance to experience the constructional principles of it with the human body. The individual stands straight with his/her feet slightly apart. Then, the knees are bended by pushing the pelvis down like sitting on a chair. The angle that the upper legs and the calf makes resembles a chair. The instructor or a group member might exert pressure on the upper legs in order to make the practitioner understand the structural system of this object during carrying a load.

20. Column/ Salamba Sarvangasana / Shoulder Stand: This pose is also resembling the column structure, like the first pose of the schedule. But, as this is a reverse pose, it should be practiced with a warmed up body. The shoulders, touching the ground, are holding the body up in a linear position by carrying the whole load. It emphasizes the importance of the spine in carrying the body load.

21. Pillar / Viparita Karani / Legs up the Wall Pose: In this pose both of the legs are kept up in the air with $90^{\circ}$ angle position to the rest of the body. Practicing it by four people by keeping the legs of all individuals together, the total figure resembles a pillar. Pillar is a powerful constructive element used in mosques and cathedrals. Because in such buildings a wide space is covered with a huge dome and there is a necessity of carrying this giant structure. In order to understand the logic that lay behind, a load is applied on the feet of an individual in this pose. Then, when the group come together a bigger load is put down on the surface of the feet of all. This little experiment helps to prove that the load bearing capacity can be increased by increasing the surface area.

22. Removing Tensile Strength of the Load-bearing System / Shavasana / Corpse Pose: In this pose all the tension of the body is cleared away by loosening and releasing it. A yoga session is generally ended with this pose. When the load-bearing function of the spine is canceled, the body can't stay stand any more. Therefore, the pose is called "corpse pose". The physical integrity of the human body can't be protected when the spine doesn't function anymore. Similarly, when the constructional system of a building is injured and loses its integrity, it can't bear the load anymore and it collapses down. The reason behind the destruction of a 
The Use of Human Body as a Medium in Architectural And Structural Education: "Yogarch" Program

building after an earthquake or another natural disaster is this loss of integrity.

\section{The Implementation Process of YogArch Workshops}

The program explained in the previous section is convenient to practice with different age groups. The workshops realized might be classified in two groups: children workshops, adult workshops for undergraduate students.

\section{YogArch workshops for children}

YogArch workshops were practiced with children aged between 6-14 years-old on several occasions. As far as some poses in the schedule are practiced with partners, the age ranges of the participants are preferred to be close to each other in each group. By this way the physical and mental perception of the members of the group might be at a similar level. Each workshop started with an introduction of the instructor, workshop process and participants. Each pose is explained before practicing. The instructor gave information about the effects on the human body and the resemblance between the human body and architectural element symbolized. After practicing, the participants explained what they felt on their body and try to explain the possible effects on a building. The workshop took approximately 60 minutes. After completing the whole schedule, the participants answered a short questionnaire. Permissions for visual recordings and data collection were taken from the parents of the participants.

Four workshops have been realized with children with a total number of 57 participants. Three of these workshops were with children aged 6-7 years old (15,11 and 8 participants in each) implemented as a part of a built environment education program realized by "Çocuk İstanbul" between 2015-2017, in İstanbul. The other one was included in a long term built environment education program called "Play Without Barriers" realized in 2014, in Bursa. The participants were aged between 8-14 years old (23 participants). The instructor determined the educational approach according to the inclination and age range of the group. During the workshops with 6-7 years old children, the explanations about the building elements and structures were less detailed. Playful explanations were added in order to attract the participants' attention. Some of the participants found the practice inspirational for their daily play routine.

The YogArch workshop realized with 8-14 years old children was followed by a clay modeling exercise. The participants designed and modeled a shelter using the constructional principles they've learned at the YogArch session. Fifteen of the total twenty-three participants found the body experience useful for designing their own constructions.

\section{YogArch workshops for undergraduate students}

Four individual YogArch workshops were practiced with the first year undergraduate architecture students of different universities in Turkey. The number of the participants of each workshop were: 64 (realized in 2014, at METU Department of Architecture), 39 (realized in 2014, at BOU Department of Architecture), 36 (realized in 2017, at AGU Department of Architecture), and 25 (realized in 2015, at TOBB ETU Department of Architecture); 164 people in total. The workshops were organized within 
architectural and structural design courses. The aim of YogArch workshop practiced with undergraduate students was to help freshmen students understand vocational terms in a practical way and reflect this knowledge to their designs. As far as it was designed to be a part of vocational education, the information given in the explanation parts during the implementation of the schedule was much more detailed compared with children workshops. The working principles of architectural elements and systems were explained on visuals supported by graphics drawn simultaneously. The participants were asked to relate the terms they learn during formal lectures with the practice they experience at YogArch. During the practice, the participants discussed the terms and working principles of architectural elements and shared their own experiences and what they felt with the other members of the group. As far as the explanations were more detailed and there were group discussions on the terms learned, the workshops for undergraduate students took a longer time compared with the workshops for children. Usually, a YogArch workshop for undergraduate students was completed in 80 minutes. At the end of each workshop all participants answered a short questionnaire about the outcomes. Also face to face interviews and group conversations were made for the evaluation of the workshop process. The visual recordings were made under the permission of the participants.

\section{FINDINGS}

The data collected from each workshops was classified according to the age group of the participants and the findings were analyzed. The methods used in the collection of this data were interviews with the participants, group conversations and short questionnaires about the participants' experience. In these questionnaires, the most successful / enlightening yoga pose-architectural element matching according to the participant was asked. Also the most distinctive quality of the program, the most effective and the most ineffective parts of the schedule were questioned. The other subjects examined in the questionnaire were the effects on their perception and the difficulties of the schedule. The participants asked to evaluate the program from useful to useless in 5 point Likert Scale.

The answer sheets of 57 children aged between 6-14 years old, who attended the workshops at four different sessions, were examined and analyzed (Figure 7). \%26 of the participants of this group found Pose-11 (Tunnel) most effective and enjoyable (Figure 8). The whole group worked as a team in this pose to form the tunnel structure. Also the playlike character of the pose made it more enjoyable for younger age groups. Each member of the group passed under the tunnel and experienced how the system works. The second most successful matching according to the members of the group was Pose-16 (Bridge) with \%21. This was an individual pose based on self-experience. Each participant explored the limits of his/her body in this pose and examined how the weight of the structure is transferred to the ground. The third favorite matching was Pose-6 (Dome) (Figure 9). \%14 of the participants of this group found this pose efficient for learning the principle laying behind. This was a smaller group pose in which 4-6 participants work together. They all came together to form the structure of a dome. It also had a play-like character, when a load was implemented on top. The members of the group worked and resisted as a team to carry the load. It was very enlightening for 
The Use of Human Body as a Medium in Architectural And Structural Education: "Yogarch" Program

understanding how the weight of the load was transferred to the ground through the dome structure. The other asana-architectural element matchings found efficient by this age group were Pose-22 (Corpse) with $\% 12$, Pose-18 (Table) with \%10, Pose-8 (Column and beam) with \%9, Pose-3 (Arch) with \%3, Pose-20 (Column) with \%3 and Pose-2 (Balcony) with $\% 2$.

Figure 7. Most effective yoga pose- architectural element matching in children \& undergraduate YogArch workshops

Figure 8. Pose-11/Tunnel pose in a YogArch workshop for children
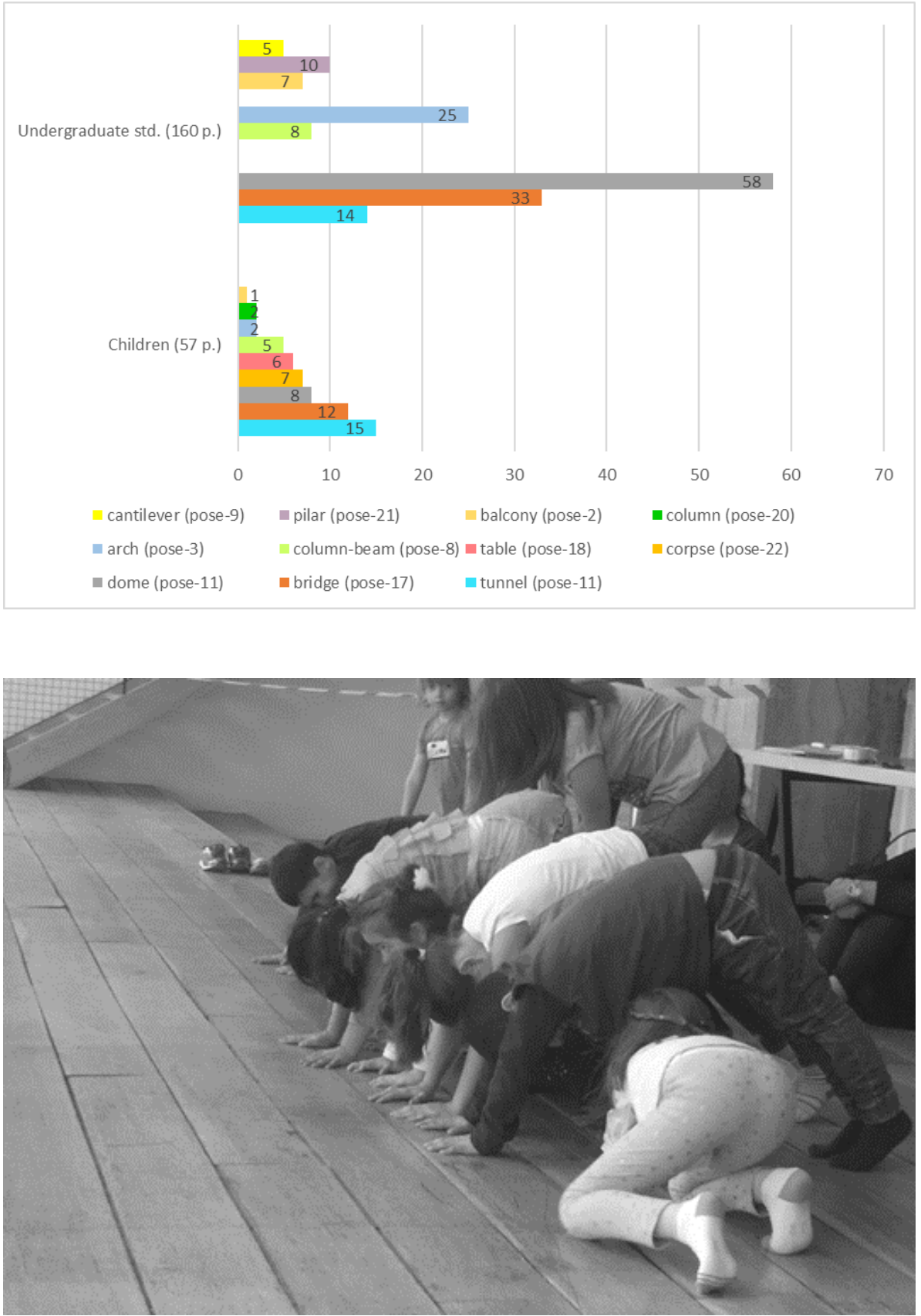


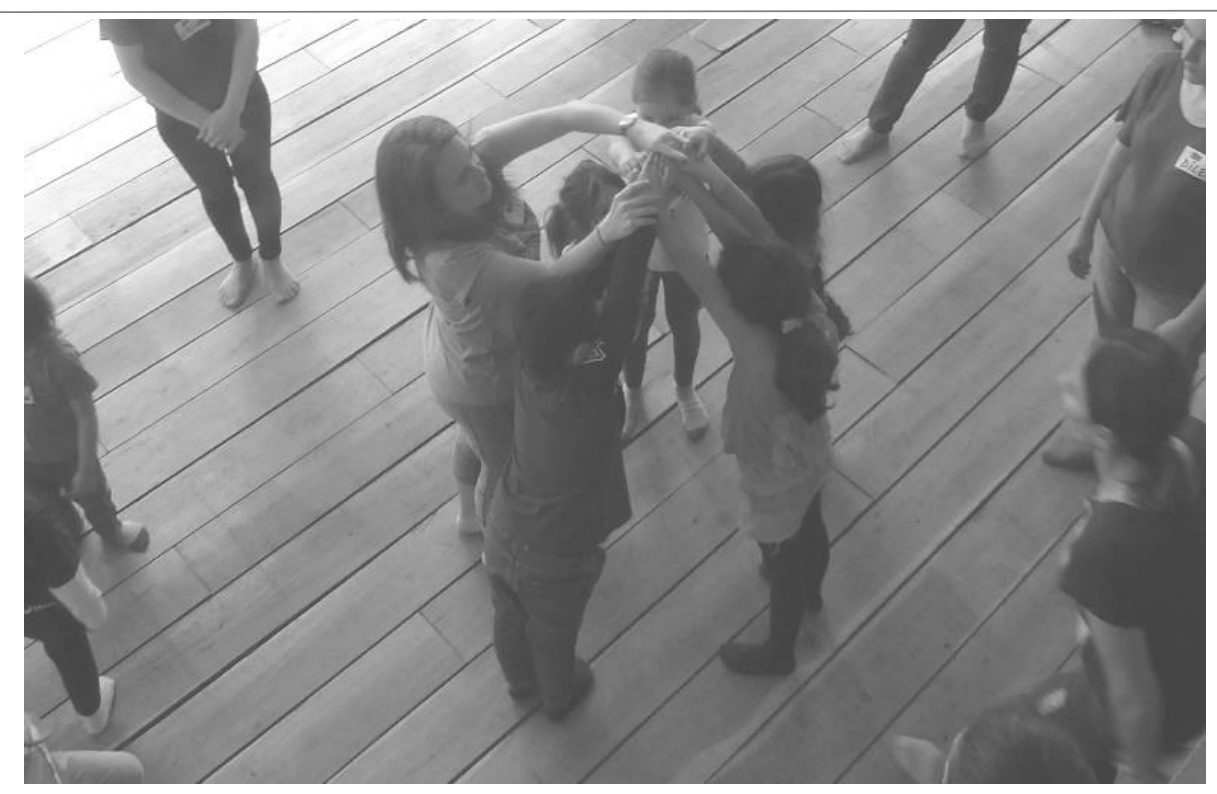

The answer sheets of 164 undergraduate students who attended the workshops from four different universities at separate sessions were examined and analyzed. \%36 of the participants of this group found Pose6 (Dome) the most effective matching. (Figure 10). In the theoretical explanation, the working principles of the dome structure and the forces effecting it were explained in detail. Depending on the physical capabilities of the participants, the whole weight of a human body might be hang on top of the dome constructed by the group members. When this pose was followed by the supported dome pose (Pose-7) the changes in the durability of the "human dome" against loads and structural forces were better understood. According to $\% 21$ percent of this group, Pose- 17 (Bridge) was successful in explaining the working principles of the structure. It is interesting that the order and percentage of this pose is the same with the younger age group's choices. \%16 of the participants of this group found Pose-3 (Arch) as a successful asana-architectural element matching (Figure 11). Two individuals came together and formed a pointed arch in this pose. The hands worked as a key-stone together. The changes in the compression and tension forces might be experienced by changing the distance between two bodies. In this participant group, the other poses found effective in explaining the structural systems were as follows: Pose-11 (Tunnel) with \%9, Pose-21 (Pillar) with \%6, Pose-8 (Column and beam) with \%5, Pose-2 (Balcony) with \%4 and Pose-9 (Cantilever) with \%3.

workshops commented that the program was most successful in explaining the concepts of durability, balance and structural mechanisms. Whereas for the undergraduate students the most clarified concepts were structural mechanism, body mechanism and structural forces (Figure 12). The different perceptions of successful concepts were caused by the difference in the implementation method of the schedule. The main motto of a children workshop was turning the YogArch schedule into a play-like activity in which participants enjoy their time and learn basic concepts and terms of architecture. In order to raise awareness on built environment and make the gained knowledge permanent, the play-like potential of yoga was used. The physical activity combined with aural and visual representations of architectural concepts turned the whole schedule into a fluent activity. Sharing this experience with their peers,
Figure 9. Pose-6 /Dome pose in a YogArch workshop for children 
The Use of Human Body as a Medium in Architectural And Structural Education: "Yogarch" Program

the children saw this activity as an opportunity to play. As far as playing is an important method of learning in pre-school and primary school education, body experience combined with peer-play become more effective for relaying information to children. The concepts of durability and balance were explained with poses in which participant interact with the instructor or the other members of the group. For example, in Pose-2 (tree pose), the instructor implemented different amounts of weight on participant's leg in order to show how the body structure reacts to the changes in the loads applied to it. Meanwhile the participant tried to resist and protect his/her balance, like a balcony in a building. Also in Pose-8 (big toe-hold), two individuals mimicked a column and beam structure. When the distance between the partners was changed the practitioners experienced the difference it made on the durability of the system. Also when the instructor implemented weight on the legs at different positions, the working principle of the structure was better understood. The interaction of the participants and the instructor enriched the play-like character of the YogArch schedule. The most appreciated pose by children in the schedule was Pose-11 (downward facing pose) in which the whole group worked together to form a tunnel. In order to deepen in the pose and keep the participants stay in the pose as long as possible some attractions were added. The members including the instructor passed under the tunnel in order to stay as long as possible in the pose and understand how the durability of the structure was provided.

Figure 10. Pose-6/Dome pose in a YogArch workshop for undergraduate students

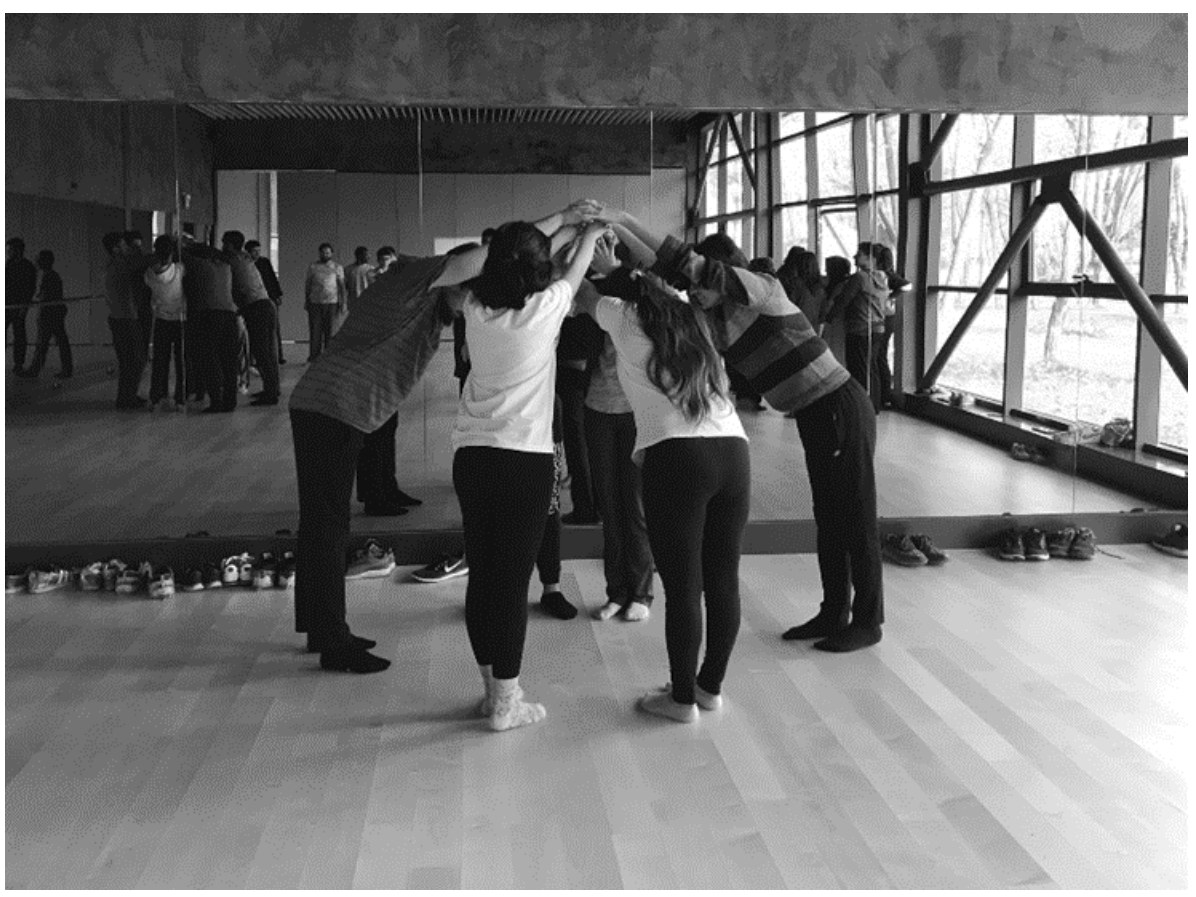



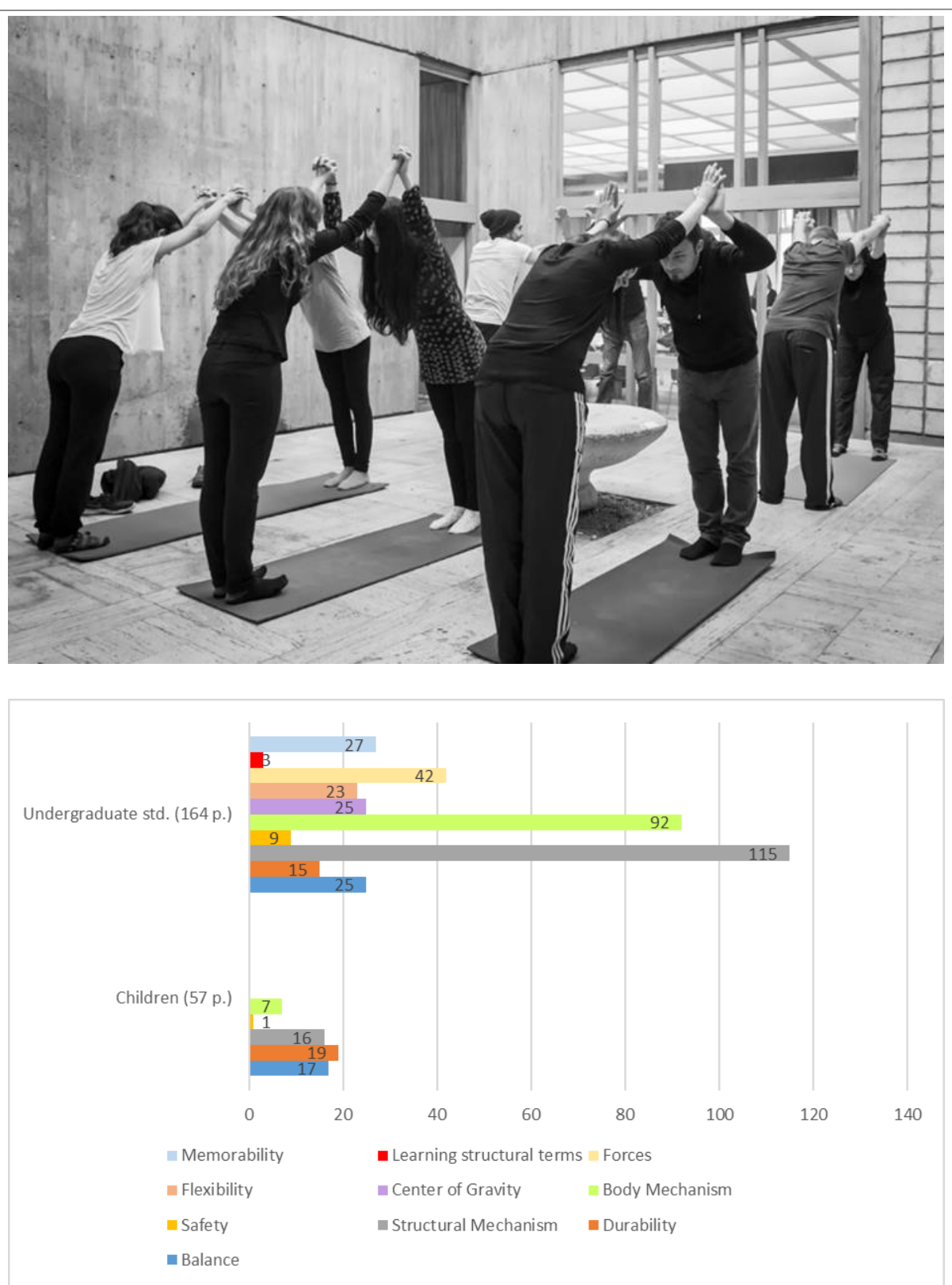

The younger participants (6-14 years-old) attended the YogArch

The answers to the questionnaires showed that the undergraduate students tended to focus on more technical concepts in the schedule. When implementing YogArch schedule with undergraduate students, vocational concerns became more determinant. The instructor emphasized on structural terms, forces effecting the constructional system when explaining each asana-architectural element match. Therefore, the participants focused on perceiving systematical principles while practicing the poses. They tried to understand both the functioning of human body and the building structure and the similarities in-between. Due to all these reasons, in this participant group, top three choices about the most clarified concepts by the schedule were structural mechanism, body mechanism and structural forces. This is an outcome of the theoretical support given to the physical activity (Figure 12).

At the end of each workshop session the participants asked to evaluate the program from useful to useless in 5 point Likert Scale. In none of the groups the program was found partly useless or useless. The younger
Figure 11. Pose-3 /Arch pose in a YogArch workshop for undergraduate students

Figure 12. Prominent concepts in YogArch workshop acquisitions 
The Use of Human Body as a Medium in Architectural And Structural Education: "Yogarch" Program

participants (6-14 years-old) attended the YogArch workshops evaluated the schedule as $\% 93$ useful, $\% 5$ partly useful and $\% 2$ neither useful nor useless (Figure 13). Besides gaining built environment awareness, this age group found this activity as a good inspiration for their daily play routine. This is remarkable to show that the concepts explained during the implementation of the program were internalized and have the potential to become permanent depending on self-repetition in daily life.

Figure 13. Prominent concepts in YogArch workshop acquisitions
Figure 14. Satisfaction level of participants in Undergraduate YogArch Workshops

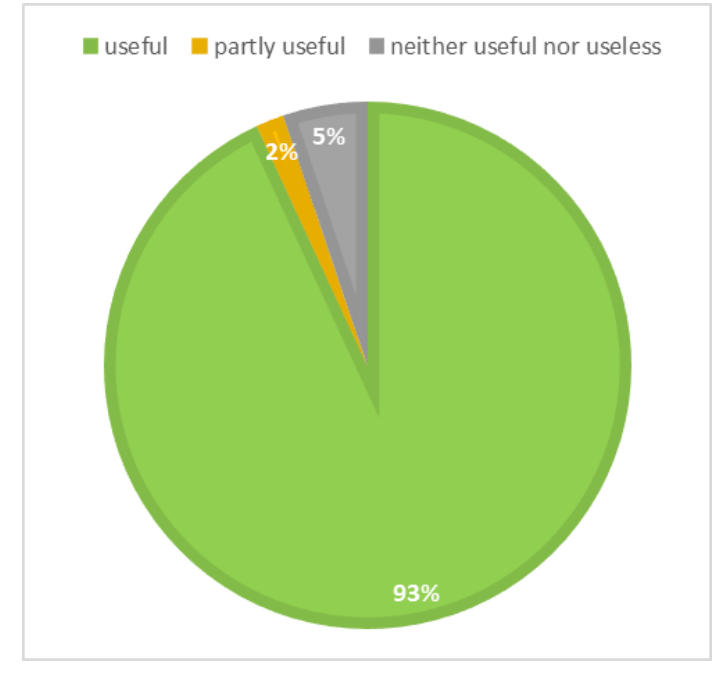

The undergraduate students attended the YogArch workshops evaluated the schedule as $\% 79$ useful, \%18 partly useful and \%3 neither useful nor useless (Figure 14). The participants commented that the implementation of the program supported and clarified the knowledge they've obtained in their formal courses. They also added that using body experience in learning constructional rules is a practical method to simplify complicated terms. This method might also be adapted to other terms and elements which are not present in the original program.

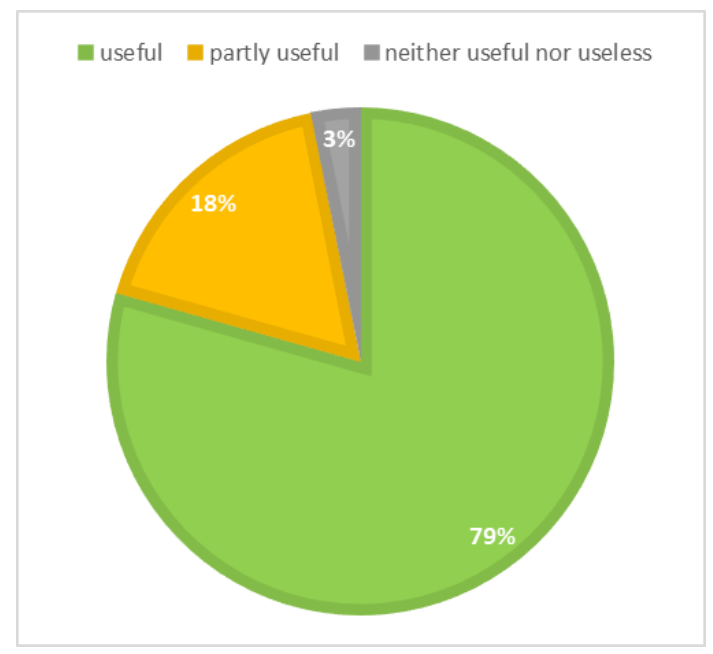

Even though, all poses used in the schedule are simple and at beginner level, some participants had difficulties due to their own lack of training, in practicing some of the poses which require reasonable amount of strength. In undergraduate workshops, $\% 23$ of the participants complained about their lack of training and stated that they had difficulties in practicing some of the poses such as Pose-9 (cantilever / 
half-moon pose), Pose-10 (mushroom building / warrior III pose), Pose17 (bridge / wheel pose) and Pose-20 (column / shoulder stand). This was the only detected weakness of the program. But in the discipline of yoga, there are a number of versions of each asana suitable for the differing ability and sufficiency of the practitioner. Therefore, if the instructor has sufficient yoga training and teaching experience, he/she might support and direct the participant to practice the pose in a more suitable version and develop body awareness.

\section{CONCLUSION \& SUGGESTIONS}

YogArch program is created in order to become a supportive educational material for built environment education (BEE). Magliocco (2003) states that in order to make "architecture", architectural education is not always an obligation, but the education of the public on built environment is a must. The quality of architectural environment is directly related with the consciousness of users as much as professionals. The safety of a living environment depends on the consciousness level of inhabitants and users. Providing sufficient knowledge on constructional principles of buildings for all members of the society and extending built environment education have vital importance especially for the countries which are under earthquake risk, like Turkey. Being aware of the risks and vulnerability of their built environment, users can be more conscious about taking the right precautions against natural disasters. One of the main reasons of designing this program is creating this consciousness for the youngsters. The earlier this awareness is gained, the more effective and permanent it becomes.

The second target group of YogArch program is the freshmen students of architecture and civic engineering. Supporting the formal curriculum based on theoretical knowledge with an informal method based on body experience makes it more understandable for the students, who are facing with the complicated academic study for the first time. Even though the YogArch schedule forms a framework with a limited number of architectural elements and rules, once the basic principle of the schedule is understood, it might be adapted to numerous examples by the users. At that point, human body would serve as a practical tool for experimenting on structural principles.

The general context of the program has the potential to be adapted to any age group. The YogArch workshops mentioned in this paper were realized with different age groups. The schedule was both used as part of built environment education for children aged between 6-14 years old, and a part of first year architectural education integrated with architectural design and basic design courses in various universities. Some suggestions might be made on the future use of the program depending on the feedback supplied from the previous implementations. In the case of YogArch workshops for children, the program might be spread to a wider community. Architecture is an interdisciplinary subject related with various areas. Consequently, there are many programs and activities realized with the aim of promoting these areas to children. In the UIA Built Environment Education Guidelines (Peck, 2002), it is stated that "critical thinking, responsible citizenship, cultural literacy, social relevance and environmental sustainability all can be addressed through using issues of the built environment to teach traditional curriculum material." The topics related with built environment and architecture are also practical tools for teaching other academic subjects such as 
The Use of Human Body as a Medium in Architectural And Structural Education: "Yogarch" Program

mathematics, literature and science. Therefore, YogArch schedule might be integrated with the curriculum of one of these lectures in primary education. Besides it might be used as a tool for raising built environment awareness as a part of project and performance tasks.

In the case of YogArch workshops for undergraduate students, the schedule might be used as a warm-up exercise for whole first year students of architecture and civic engineering. By this way the freshmen students might easily internalize basic technical terms that they would be dealing with in their whole vocational education. Besides, as far as the program is based on group working, it might create a social atmosphere for the freshmen students. The schedule might also be implemented in the scope of architectural design studio as a primary exercise before designing the first constructional entity. Experiencing the structural principles would be useful for the students who have limited structural knowledge and who are expected to create a design with constructional integrity. It would also be effective to use YogArch program as a part of structural design or statics and strength lectures. It would be beneficial to simultaneously experience the structural forces, which are theoretically explained in the lecture, on their own body.

Figure 15. The interactional cycle between "human body" and "architecture Satisfaction level of participants in Undergraduate YogArch Workshops
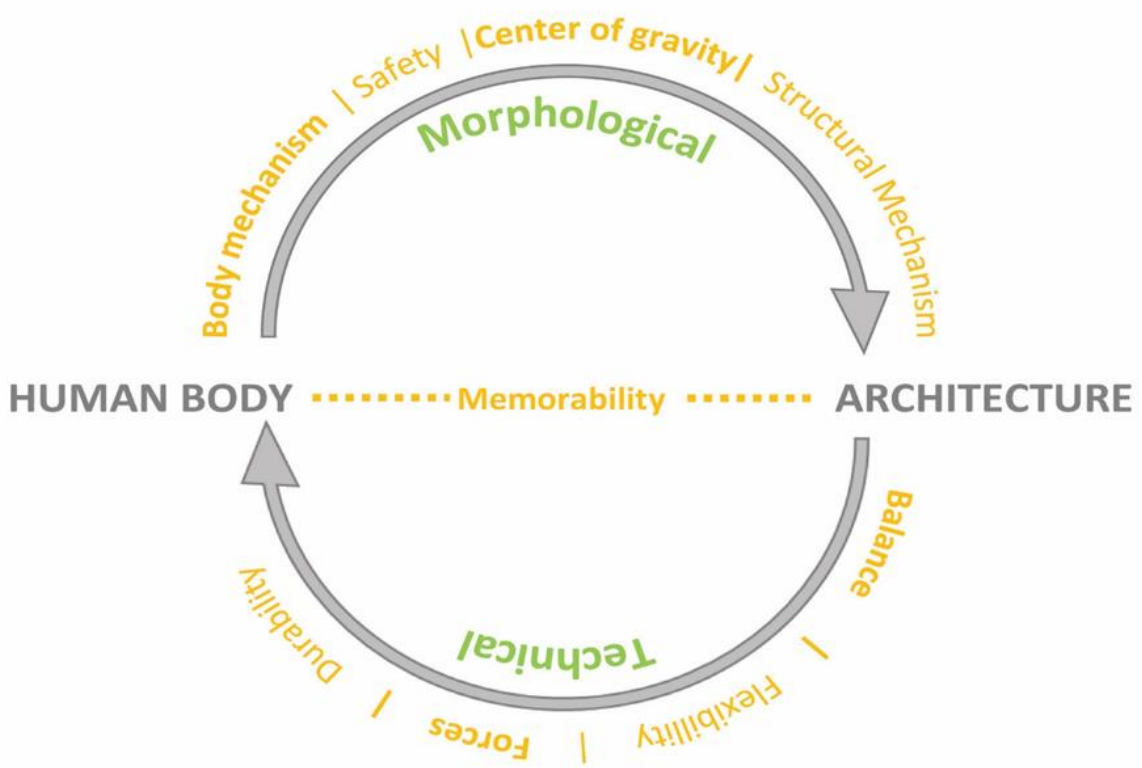

The previous researches in the literature, which are mentioned in the scope of this paper, claim that there are morphological and technical connotations between human body and architecture. Architects have been inspired from human body in both terms. The findings of YogArch workshops indicate that the correlation between human body and architecture might also be used in analyzing and decoding architecture. In other words, the source of inspiration might be used as the key of understanding and internalizing. The participants of YogArch workshops expressed that the program eased the perception of morphological and technical interactions between human body and architecture. The prominent concepts in the participants' comments on their selfexperience show that YogArch serves as a medium for revealing the resemblances between body and structural systems. Also the use of human body enhances the memorability of the knowledge and experience acquired (Figure 15). 
This program which integrates body experience and architectural knowledge has the potential to be adapted to different occasions. Due to the fact that the physical aspect of yoga is suitable for all ages, the YogArch program is also applicable with participants of various ages. Due to the play-like character of the schedule, the participants might spend an enjoyable time learning and thinking about architectural and structural rules. This process makes the gained information more permanent.

Since the beginning of architectural history, human body has been seen as a source of inspiration for architectural design. The proportions of human body, the mechanism of the biological entity of the human body guided architects for solving constructional problems in their designs, for centuries. Depending on this fact, reading this equation in reverse and using human body for understanding constructional systems emerge as a reliable method.

\section{ACKNOWLEDGEMENTS}

We would like to thank the participants of "YogArch" workshops who attended on voluntary basis, including the workshops realized at "Çocuk İstanbul", PWB project (children sessions) and first year students of Faculties of Architecture at BOU, METU, AGU and TOBB ETU (undergraduate sessions). The workshops mentioned in the paper have been spread over a wide time span from 2013 to 2017. The data collection and visual recordings are made with the permission of the participants. Unless otherwise stated, all rights of the tables, images and photos belong to the author.

\section{FINANCIAL DISCLOSURE}

The author declared that this study has received no financial support.

\section{ETHICS COMMITTEE APPROVAL}

Ethics committee approval was not required for this article.

\section{LEGAL PUBLIC/PRIVATE PERMISSIONS}

In this research, the necessary permissions were obtained from the relevant participants (individuals, institutions and organizations) during the questionnaires, observation and experiment.

\section{REFERENCES}

Alberti, L. B. (1988). On the Art of Building in Ten Books. Cambridge, Mass: MIT Press.

Arın, S. (2013). Architectural Education For Children; A Case Study: Experiencing Architecture By Yoga. International Workshop COAC Barcelona 2013. 29-31 May 2013. Universitat Politécnico de Catalunya: Barcelona; (ISBN 978-84-695-9424-7) (http://www.pa.upc.edu/Va ris/altres/arqs/congresos/international-workshop-coac-barcelona-201 3-jornadas-cientificas-coac-barcelona-2013/comunicaciones-isbn-in-pr ocess/Sebla\%20Arin.docx/view) (Accessed on 10.04.2020) 
The Use of Human Body as a Medium in Architectural And Structural Education: "Yogarch" Program

Chiou S, Chiu W. (2010). On design methodology and practice of bionics. In: Proceedings of the 10th IEEE international conference on digital game and intelligent toy enhanced learning. Kaohsiung, Taiwan

Gruber, P. (2011). Biomimetics in Architecture: Architecture of Life and Buildings. Wien: Springer Pub.

Kaminoff, L. \& Matthews, A. (2012). Yoga Anatomi. İstanbul: Sistem Yay.

Knudson, D. (2007). Fundamentals of Biomechanics. Chico, California: Springer Pub.

Le Corbusier. (2014). Modulor. İstanbul: YEM Yay.

Lotfabadi, P., Alibaba, H. Z. \& Arfaei, A. (2016). Sustainability; As a combination of parametric patterns and bionic strategies. Renewable and Sustainable Energy Reviews. V:57, pp: 1337-1346.

Magliocco, A. (2003). "Education in Architecture. University role into architectural-environmental education: parallel experiences of didactic workshop." EAAE Transaction on architectural education $\mathrm{N}^{\circ} 15$ Writings in architectural education, EAAE prize 2001-02 (ed. Ebbe Harder), Royal Danish Academy of Fine Arts, School of Architecture, Copenhagen: 207217.

Pappas, S. (2006). Yoga Posture Adjustments and Assisting. Victoria,BC: Trafford Pub.

Peck, M. (Dir.). (2002). UIA Built Environment Guidelines, UIA International Work Programme "Architecture and Children (Built Environment Education).

Plowright, P. (2018). Extending Skin. ARCC Conference Repository. https://doi.org/10.17831/rep:arcc\%y552 (Accessed on 10.07.2020)

Steadman, P. (1979). The Evolution of Designs: Biological analogy in architecture and the applied arts. Cambridge: Cambridge University Press

Vidler, A. (1990). The Building in Pain: The Body And Architecture in Post-modern Culture. AA Files, No:19 (Spring 1990), pp. 3-10. https://www.jstor.org/stable/29543679 (Accessed on 019.03.2020)

Vitruvius, P. (1960). Vitruvius: the ten books on architecture. New York: Dover Pub.

Worby, C. (2007). Her Yönüyle Yoga. Ankara: Arkadaş Yay.

Url-1: $\quad$ http://figuredrawingdotinfo.blogspot.com/2015/08/whyshould-we-care-about construction_14.html (Accessed on 20.06.2020) 
Url-2: $\quad$ http://letteraturaartistica.blogspot.com/2014/01/englishversion-francesco-di-giorgio.html (Accessed on 25.06.2020)

Url-3: http://letteraturaartistica.blogspot.com/2013/11/massimomussini-francesco-di-giorgio-e.html (Accessed on 25.06.2020)

Url-4: https://www.thetimes.co.uk/article/vitruvian-man-must-stay-inhis-vault-9x527hkk8 (Accessed on 25.06.2020)

Url-5: https://www.archdaily.com/902597/on-the-dislocation-of-thebody-in-architecture-le-corbusiers-modulor (Accessed on 14.07.2020)

\section{Resume}

Sebla ARIN ENSARIOĞLU received her undergraduate and graduate degrees from ITU Department of Architecture. Her research areas are architectural design, design education, participatory architecture, children and architecture. She continues her studies as an architect since 2004, as an academician since 2012 and recently works as a member of Bursa Uludağ University, Faculty of Architecture. 